\title{
Surface roughness modeling of semi solid aluminum milling by fuzzy logic
}

\author{
Savkovic B. ${ }^{1}$, Kovac P. ${ }^{2 *}$, Mankova I. ${ }^{3}$, Gostimirovic M. ${ }^{4}$, Rokosz K. ${ }^{5}$, Rodic D. ${ }^{6}$ \\ 1, 2, 4, 6 Faculty of Technical Sciences, University of Novi Sad, Novi Sad, Serbia \\ ${ }^{3}$ Faculty of Mechanical Engineering, Technical University of Kǒsice, Kǒsice, Slovakia \\ ${ }^{5}$ Koszalin University of Technology, Koszalin, Poland
}

\begin{abstract}
Index Terms
Face Milling

Surface Roughness

Factorial Plan

Fuzzy Logic

Modeling

Abstract - In the paper carried out was modeling of cutting parameters in face milling process of Semi Solid Metal alloys. As input parameters in the process of modeling were taken: cutting speed v, the feed per tooth and depth of cut, while for the output characteristics of the process were taken arithmetic mean surface roughness $\mathrm{Ra}$ and maximum roughness height $\mathrm{R}_{\max }$. Modeling was done in two ways. The first model was made with the help of mathematical and statistical method-factorial experiment DoE, where it was used model with parameters' interaction. The second model was made by artificial intelligence and as a tool was chosen fuzzy logic.
\end{abstract}

Received: 14 October 2016

Accepted: 5 January 2017

Published: 21 April 2017

\section{INTRODUCTION}

The rapid industrial progress in parallel with the development of science, has created a basis for the further development of the science of machining. Different techniques of artificial intelligence, from expert systems, artificial neural networks, fuzzy logic, genetic algorithms and genetic programming and other modeling techniques find their application in this area.

This work aims to show the possibility of applying mathematical modeling based on factorial experiment DoE and fuzzy logic in milling, as one of the most common machining processes. There is a need to improve machining processes by applying advanced modeling techniques, or simulation, which also includes the modeling by using the methods of artificial intelligence. The resultant models are used for the analysis, management and selection of optimal process parameters, which represent the image of the complex relationships lost between the input and output
(C) 2017 The Author(s). Published by TAF Publishing.

\footnotetext{
* Corresponding author: Kovac P.

†Email: pkovac@uns.ac.rs
}

parameters of milling process. The resulting models can be utilized with sufficient accuracy in the adaptive management and monitoring of the process and making decisions in real time. This is of great importance in the exploitation of intelligent manufacturing systems. Also it is possible to optimize the input parameters of the process on the basis of setting the machining limits, and in order to achieve one or more target functions such as reducing cutting force and/or minimization of surface roughness, which from a technical standpoint are with the highest practical value and meaning.

\section{A. Modeling Processes Using Multi-Factor Experiment Plan-DoE}

By the model are expressed the essential characteristics of an object, process or system. The mathematical model consists of a system of equations, conditions and algorithmic rules. 
The most widely used models of multifactor experiments in which the factors vary in two levels (maximum and minimum value), with the mean value of factors which is not treated as a level variation. This type of experimental plan needs next experimental points:

$$
N=2^{k}+N_{0}
$$

\section{B. Three factorial second order plans}

Possibility of composing these plans can be seen in Figure 1. The first cycle usually departs from the model of simple shapes and orthogonal plans of lower rank. Then continues if it finds out this model to be inadequate in the second cycle and takes the model with mutual influences. Since in the multifactorial between the plans of the first and higher order interconnection exists, plans are going to compose higher order model, so that the whole set of existing experimental points is used in the plan of a higher order. It remains then only to carry out supplementary experiments in accordance with the plans of the second order. In this way, the continuity of the research goes on through successive cycles. Also in this way are achieved lower costs and shorter time of the survey.

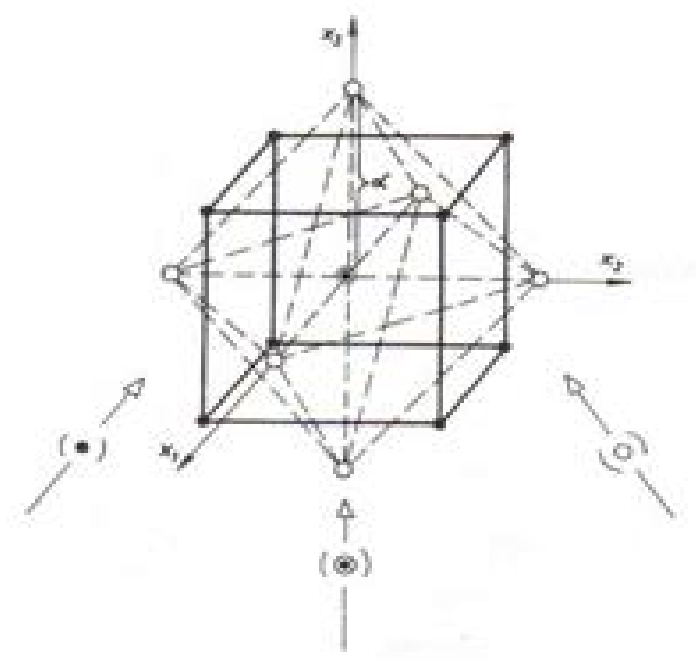

Fig. 1. The possibility of composing second order plans [5]

\section{The Application of Artificial Intelligence in Process}

According to the structure and management issues new production has little in common from the 50s of the last century. Based on which is the present production as market demand, is new production philosophy that is based on knowledge, advanced manufacturing and information technology, new materials and advanced machining and manufacturing systems. The biggest changes in technological development in the history of mankind took place during last fifty years due to the rapid development of technology and computers. A special role in the automation and improvement of production processes has artificial intelligence.

\section{Fuzzy Logic}

Fuzzy logic (FL) is a generalization of the classical Boolean (Boolean) logic, a theory on it and stage sets designed phase systems can be viewed as a generalization of conventional expert systems based on rules. Fuzzy systems manifest symbolic and numerical characteristics.

It can also be said that fuzzy logic and fuzzy inference systems are an effective technique for the identification and control of complex nonlinear systems. Fuzzy logic is also used for prediction. The theory of fuzzy logic, initiated by [1], was primarily beneficial to work with uncertain and vague information. Fuzzy logic is particularly attractive because of its ability to solve problems in the absence of precise mathematical models. This theory has proven to be an effective means for facing with the objectives that are set out in the reeds linguistic terms, such as "small", "medium" and "high," which can be defined as fuzzy sets [2].

Fuzzy inference systems in the literature can be found under different names depending on the method of application: as a system based on fuzzy rules, stage designs, stage associative memory (FAM) or phase controllers (when used for the management of a system). Basically, the phase inference system is composed of five functional blocks [3]:

- Rule base contains a number of if-then rules for the stage, - Database that defines the membership function of input / output variables that are used in the phase rules,

- Decision-taking unit that performs the operation of reasoning,

Fuzzification interface that transforms crispbread entrance to the degree of membership of a particular linguistic value,

- Defuzzification interface that is the result of the conclusion of the stage transforms into a crisp output. Very often, the rule base and database are identified together as a knowledge base. Concluding phase diagram of the system is shown in Figure 2. 


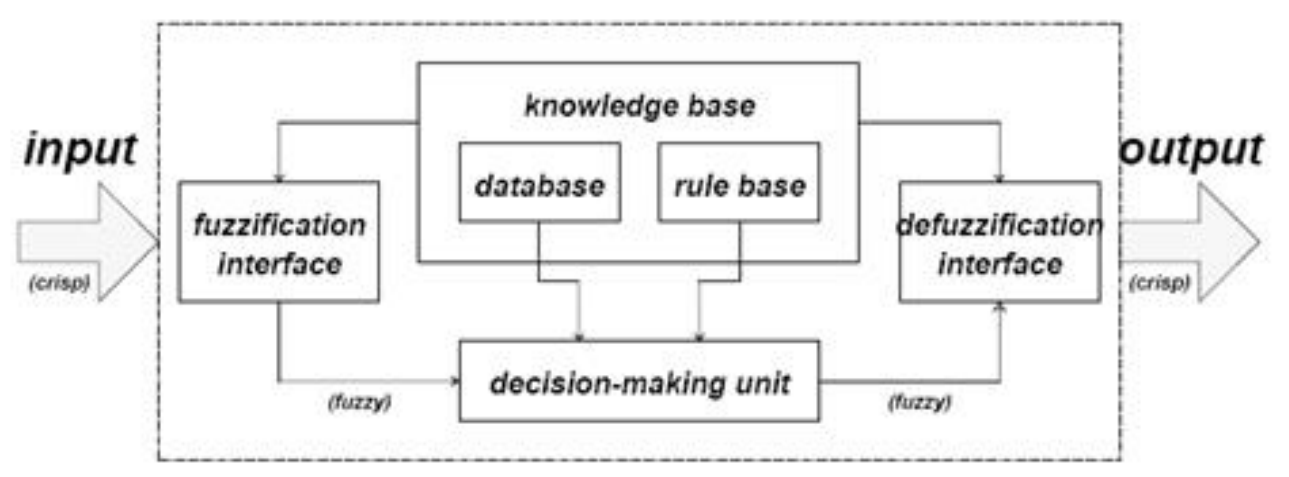

Fig. 2. Fuzzy inference system [9]

\section{DESCRIPTION OF EXPERIMENTS IN FACE MILLING}

\section{A. The Material of the Workpiece}

The experiments were carried out on aluminum alloy 7075 (Al-Zn-Mg-Cu), which was obtained by the new method of casting, or SSM process. The width and length of the workpiece was $100 \mathrm{~mm}$, and the thickness was $15 \mathrm{~mm}$. Chemical composition and mechanical properties of alloy 7075 are given in Table 1 and Table 2. The alloy 7075 which is examined in this paper was not thermally treated, but its characteristics are examined immediately after casting.

TABLE 1

Chemical composition of aluminum alloy 7075

\begin{tabular}{llllllllll}
\hline \hline Basic designation & Basic element & $\mathrm{Zn}$ & $\mathrm{Mg}$ & $\mathrm{Cu}$ & $\mathrm{Cr}$ & $\mathrm{Fe}$ & $\mathrm{Si}$ & $\mathrm{Mn}$ & $\mathrm{Ti}$ \\
\hline 7075 & $\mathrm{Al}$ & 5.8 & 2.52 & 1.65 & 0.2 & 0.18 & 0.1 & 0.025 & 0.025 \\
\hline \hline
\end{tabular}

TABLE 2

Mechanical properties of aluminum alloy 7075 in the cast condition

\begin{tabular}{llll}
\hline \hline Type of alloy & Tensile strength $\mathrm{R}_{m}[\mathrm{MPa}]$ & Yield strength $\mathrm{R}_{p} 0.2[\mathrm{MPa}]$ & Elongation [\%] \\
\hline $7075-0$ & 262 & 103 & 17 \\
\hline \hline
\end{tabular}

\section{B. Machine Tool and Cutting Tool}

The machine on which procedures were performed is a vertical milling machine "PRVOMAJSKA"" FSS-GVK -3. During testing was used for face milling head "JUGOALAT" G.037 $100 \mathrm{~mm}$, with mechanically fastened cutting inserts of K20 quality, with the following characteristics:

- the number of teeth $\mathrm{z}=5$,

- incidence angle $k=75^{\circ}$,

- face angle $Y=0^{\circ}$.

\section{The Device for Measuring Surface Roughness}

Modern surface roughness gauges are used for measuring the surface roughness of the object [4]. Used device like "MARSURF PS1" to measure the maximum roughness height Rmax, and the arithmetic mean roughness $\mathrm{Ra}$ in microns.

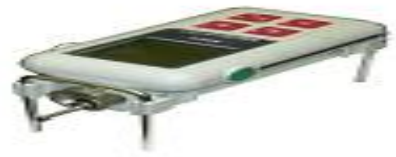

Fig. 3. "MarSurf PS1"

\section{The Machining Parameters}

Machining regime includes the following elements:

- cutting speed - $\mathrm{v}$, and the appropriate number of revolutions on the machine $\mathrm{n}$;

- feed per tooth - s1, and the appropriate speed of the milling table (s), 
so that: $\mathrm{s}=\mathrm{s} 1-\mathrm{z}-\mathrm{n}$ the number of teeth in the process was $\mathrm{z}=1$; $\bullet$ a cutting depth $-\mathrm{a}$.

The values of the machining parameters were adopted on the basis of recommendations from the literature and are presented in Table 3.

TABLE 3

Levels of experiment parameters in face milling

\begin{tabular}{|c|c|c|c|c|c|c|}
\hline Levels (Membership functions) & & Cutting speed $\mathrm{v}, \mathrm{m} / \mathrm{min}$ & Cutting speed $\mathrm{v}, \mathrm{m} / \mathrm{s}$ & The feed per tooth $\mathrm{s} 1, \mathrm{~mm} / \mathrm{t}$ & Cutting depth a, mm & Number of Rev. n, o/min \\
\hline Higher & +1.41 & 351,86 & 5,864 & 0,223 & 2,6 & 1120 \\
\hline High & +1 & 282,74 & 4,712 & 0,177 & 1,72 & 900 \\
\hline Medium & $\mathbf{0}$ & 223,05 & 3,717 & 0,141 & 1,14 & 710 \\
\hline Low & 1 & 175,93 & 2,932 & 0,112 & 0,75 & 560 \\
\hline Lower & -1.41 & 141,37 & 2,356 & 0,089 & 0,5 & 450 \\
\hline
\end{tabular}

TABLE 4

Cumulative experimental results needed to model factorial plan of experiments

\begin{tabular}{|c|c|c|c|c|c|}
\hline Ordinal number & & Factor & & & Measured surface roughness \\
\hline & $\mathrm{v}, \mathrm{m} / \mathrm{s}$ & $\mathrm{s} 1, \mathrm{~mm} / \mathrm{t}$ & $\mathrm{a}, \mathrm{mm}$ & $\mathrm{Ra}, \mu \mathrm{m}$ & $\mathrm{Rmax}, \mu \mathrm{m}$ \\
\hline 1 & 2,93 & 0,112 & 0,75 & 0,657 & 5,07 \\
\hline 2 & 4,71 & 0,112 & 0,75 & 0,666 & 4,51 \\
\hline 3 & 2,93 & 0,177 & 0,75 & 0,909 & 6,89 \\
\hline 4 & 4,71 & 0,177 & 0,75 & 0,815 & 7,97 \\
\hline 5 & 2,93 & 0,112 & 1,72 & 0,765 & 5,63 \\
\hline 6 & 4,71 & 0,112 & 1,72 & 0,472 & 4,12 \\
\hline 7 & 2,93 & 0,177 & 1,72 & 0,869 & 6,63 \\
\hline 8 & 4,71 & 0,177 & 1,72 & 0,632 & 5,78 \\
\hline 9 & 3,71 & 0,141 & 1,14 & 0,621 & 5,16 \\
\hline 10 & 3,71 & 0,141 & 1,14 & 0,553 & 4,91 \\
\hline 11 & 3,71 & 0,141 & 1,14 & 0,643 & 5,02 \\
\hline 12 & 3,71 & 0,141 & 1,14 & 0,604 & 6,3 \\
\hline 13 & 2,35 & 0,141 & 1,14 & 0,758 & 5,76 \\
\hline 14 & 5,86 & 0,141 & 1,14 & 0,482 & 4,3 \\
\hline 15 & 3,71 & 0,089 & 1,14 & 0,568 & 3,34 \\
\hline 16 & 3,71 & 0,223 & 1,14 & 1,07 & 7,77 \\
\hline 17 & 3,71 & 0,141 & 0,5 & 0,818 & 5,31 \\
\hline 18 & 3,71 & 0,141 & 2,6 & 0,921 & 6,83 \\
\hline 19 & 2,35 & 0,141 & 1,14 & 0,774 & 6,11 \\
\hline 20 & 5,86 & 0,141 & 1,14 & 0,528 & 4,63 \\
\hline 21 & 3,71 & 0,089 & 1,14 & 0,68 & 3,45 \\
\hline 22 & 3,71 & 0,223 & 1,14 & 1,026 & 7,47 \\
\hline 23 & 3,71 & 0,141 & 0,5 & 0,775 & 5,66 \\
\hline 24 & 3,71 & 0,141 & 2,6 & 1,093 & 6,3 \\
\hline
\end{tabular}

During the measurement, surface roughness obtained were records of over 20 roughness parameters, but in the study were used the mean roughness ( $\mathrm{Ra}$ ) and the maximum roughness height (Rmax). Figure 4 shows an ex- ample of the measured roughness of the machined surface profile, obtained by means of the device for measuring the roughness "MarSurf". Overview of the measured surface roughness is shown in the summary Table 4. 


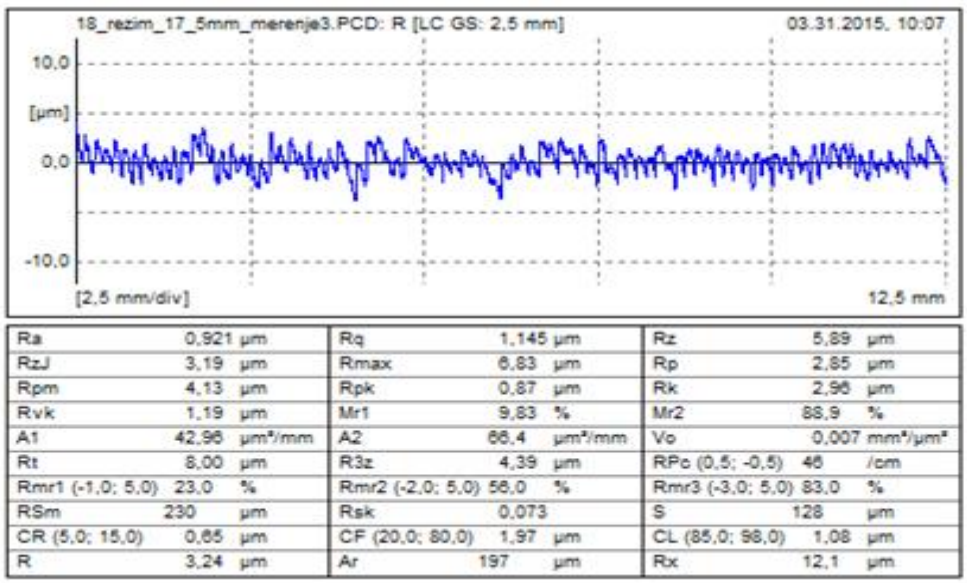

Fig. 4. The results of the measured roughness profile of machined surface

TABLE 5

Measured and calculated values

\begin{tabular}{|c|c|c|c|c|}
\hline Ordinal number & Measured values & Calculated values & Measured values & Calculated values \\
\hline & $\begin{array}{l}\text { Mean. arithm. } \\
\text { roughness } \mathrm{Ra}, \mu m\end{array}$ & $\begin{array}{l}\text { Model with } \\
\text { interaction of }\end{array}$ & $\begin{array}{l}\text { Maximum roughness } \\
\mathrm{R}_{(\max ,)} \mu m\end{array}$ & $\begin{array}{l}\text { Model with } \\
\text { interaction of }\end{array}$ \\
\hline 1 & 0,657 & 0,92 & 5,07 & 5,62 \\
\hline 2 & 0,666 & 0,83 & 4,51 & 4,87 \\
\hline 3 & 0,909 & 1,26 & 6,89 & 8,75 \\
\hline 4 & 0,815 & 1,17 & 7,97 & 9,43 \\
\hline 5 & 0,765 & 0,46 & 5,63 & 3,80 \\
\hline 6 & 0,472 & 0,29 & 4,12 & 2,59 \\
\hline 7 & 0,869 & 0,59 & 6,63 & 4,91 \\
\hline 8 & 0,632 & 0,39 & 5,78 & 4,17 \\
\hline 9 & 0,621 & 0,56 & 5,16 & 4,75 \\
\hline 10 & 0,553 & 0,56 & 4,91 & 4,75 \\
\hline 11 & 0,643 & 0,56 & 5,02 & 4,75 \\
\hline 12 & 0,604 & 0,56 & 6,3 & 4,75 \\
\hline 13 & 0,758 & 0,59 & 5,76 & 5,50 \\
\hline 14 & 0,482 & 0,36 & 4,3 & 4,09 \\
\hline 15 & 0,568 & 0,57 & 3,34 & 2,93 \\
\hline 16 & 1,07 & 1,04 & 7,77 & 7,32 \\
\hline 17 & 0,818 & 2,28 & 5,31 & 11,65 \\
\hline 18 & 0,921 & 0,38 & 6,83 & 3,53 \\
\hline 19 & 0,774 & 0,59 & 6,11 & 5,50 \\
\hline 20 & 0,528 & 0,36 & 4,63 & 4,09 \\
\hline 21 & 0,68 & 0,57 & 3,45 & 2,93 \\
\hline 22 & 1,026 & 1,04 & 7,47 & 7,32 \\
\hline 23 & 0,775 & 2,28 & 5,66 & 11,65 \\
\hline 24 & 1,093 & 0,38 & 6,3 & 3,53 \\
\hline
\end{tabular}




\section{E. Model of Three Factorial Experimental Plan for Face Milling}

Processing of data according the three factorial experimental plan of first level is performed for the arithmetic mean roughness $\mathrm{Ra}$ and the maximum height roughness Rmax. For easier processing of data, the Microsoft Excel software package was used. Worked out was model with interaction of parameters. The general form of second order model equations (2) is shown as follows:

The given equation is marked with the R output characteristic of machinability of milling process, while the parameters indicated by $\mathrm{F}$ are the following factors:
$\mathrm{F}_{1}$ - a cutting speed, $\mathrm{mm}$

$\mathrm{F}_{2}$ - feed per tooth $\mathrm{s} 1, \mathrm{~mm} / \mathrm{t}$

$\mathrm{F}_{3}$ - a cutting depth, mm

Three factorial model of the second order for the arithmetic mean surface roughness (3) the maximum height surface roughness (4) in face milling, depending on the cutting speed (v), feed per tooth (s1) and depth of cut (a) are in the following forms:

In Table 5 are given the measured and calculated values for the experimental model of the second order. Table 6 presents the results of the assessment of significance and adequacy of the model.

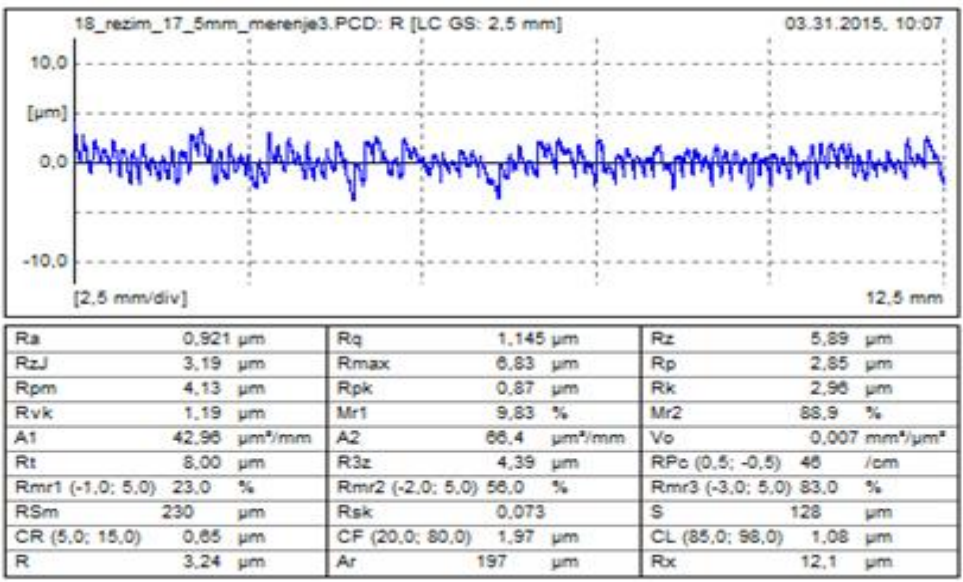

Fig. 5. The results of the measured roughness profile of machined surface

TABLE 6

ASSESSMENT OF THE SIGNIFICANCE AND APPROPRIATENESS OF MODEL

\begin{tabular}{|c|c|c|c|c|c|c|c|c|}
\hline \multirow[t]{3}{*}{ Model } & \multicolumn{4}{|c|}{ Coefficients $\mathrm{R}_{a}, \mu \mathrm{m}$} & \multicolumn{4}{|c|}{ Coefficients $R_{\max }$} \\
\hline & \multicolumn{2}{|c|}{ Regression } & \multicolumn{2}{|c|}{ Significance } & \multicolumn{2}{|c|}{ Regression } & \multicolumn{2}{|l|}{ Significance } \\
\hline & Designation & Values & Designation & Values & Designation & Values & Designation & Values \\
\hline & $\mathrm{C}$ & 257,8408 & Fr0 & 92,47 & $\mathrm{C}$ & 2,6926 & Fr0 & 1304,99 \\
\hline & $\mathrm{p} 1$ & 2,10699 & Fr1 & 26,97 & $\mathrm{p} 1$ & 1,75517 & Fr1 & 9,33 \\
\hline & p2 & 6,54356 & Fr2 & 36,68 & p2 & $-0,7356$ & Fr2 & 83,28 \\
\hline & p3 & $-0,38070$ & Fr3 & $0,17^{*}$ & p3 & $-1,0092$ & Fr3 & $0,16^{*}$ \\
\hline & p11 & $-0,89614$ & Fr11 & $3,06^{*}$ & p11 & $-0,0099$ & Fr11 & $0,00 *$ \\
\hline & p22 & 1,52763 & Fr22 & 7,67 & p22 & $-0,1217$ & Fr22 & $0,05^{*}$ \\
\hline & p33 & 0,76115 & Fr33 & 20,61 & p33 & 0,44157 & Fr33 & 6,89 \\
\hline & $\mathrm{p} 12$ & 0,09593 & Fr12 & $0,02^{*}$ & $\mathrm{p} 12$ & 1,00744 & Fr12 & $2,38^{*}$ \\
\hline & $\mathrm{p} 13$ & $-0,89570$ & Fr13 & $6,22^{*}$ & $\mathrm{p} 13$ & $-0,6067$ & Fr13 & $2,83^{*}$ \\
\hline & p23 & $-0,14108$ & Fr23 & $0,14^{*}$ & p23 & $-0,4924$ & Fr23 & $1,74^{*}$ \\
\hline & The adequacy & & $\mathrm{Fa}$ & 1,53569 & & The adequacy & $\mathrm{Fa}$ & 0,80481 \\
\hline
\end{tabular}




\section{F. Implementation of Fuzzy Logic Model-"Mamdani" Type}

Fuzzy inference system consists of five windows, three of which are used for the editing phase of the conclud- ing system. These are the concluding phase system editor, editor of membership functions and rules editor. While the remaining two viewer rules and graphical display solutions, are used only for observation regulated system, Figure 5.

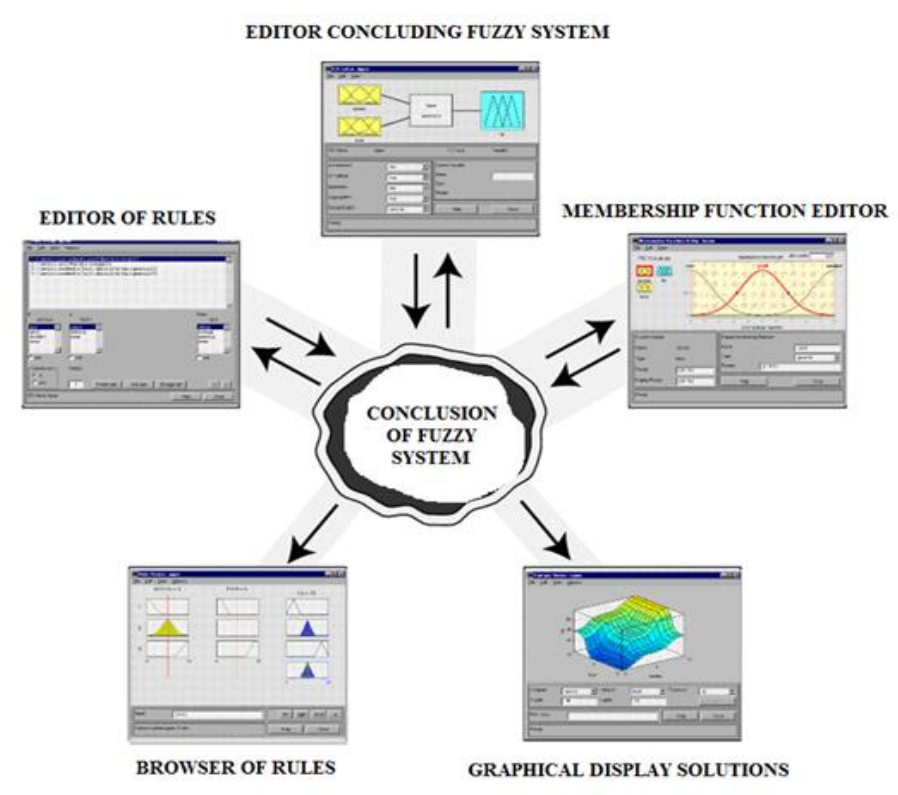

Fig. 6. Fuzzy inference system [7]

\section{G. FIS-Fuzzy Inference System}

The main role of FIS editor is to define the number of inputs, names of input and output variables, choose the type of inference (Mamdani or Sugeno). Mamdani type implies the linguistic value of the output variable phase regular meetings. Selection stage regulator Sugeno type implies the linguistic value of the output variable fuzzy sets of type singleton.

After the launch phase of the system, the screen will appear FIS Editor Window with one entrance (code input1) and one output (code input2). In the terms of reference, there are three input variables (speed, travel and depth of cut) and two output variables (Ra, Rmax). In the next step, it is necessary to define the appropriate experimental order of membership function of the measured values of the output characteristics of the machining process.

\section{H. MFE-Membership Function Editor}

MFE is a tool that allows you to view and edit all the membership functions, input and output variables for the concluding phase of the entire system. After defining the variable phase system, followed by the definition of membership functions for each variable, MFE can be opened in two ways. The first is by writing commands in the command line mfedit, otherwise using the commands in the dropdown menu FIS editor-a, by selecting Edit> Membership Functions. Before you define the membership functions for variables, existing (by default) membership functions must be deleted using the command Edit> Remove All MFS.

Types of membership functions in the capital appear in parametric form. They may be: triangular (trimf), trapezoidal (trapmf), bell (gbellmf), Gauss (gaussmf), double Gauss (gauss2mf), sigmoid (sigmf) etc.

Chosen is gaussmf membership function (5), which is divided into 5 different levels: Lower, Low, Medium, High, Higher. Lower replaced the number -1.41,

Low number of -1 ,

Medium number 0 ,

High number 1 and

Higher number of 1.41.

$$
f(x, \sigma, e)=e^{\frac{-(x-c)^{2}}{2 \sigma^{2}}}
$$




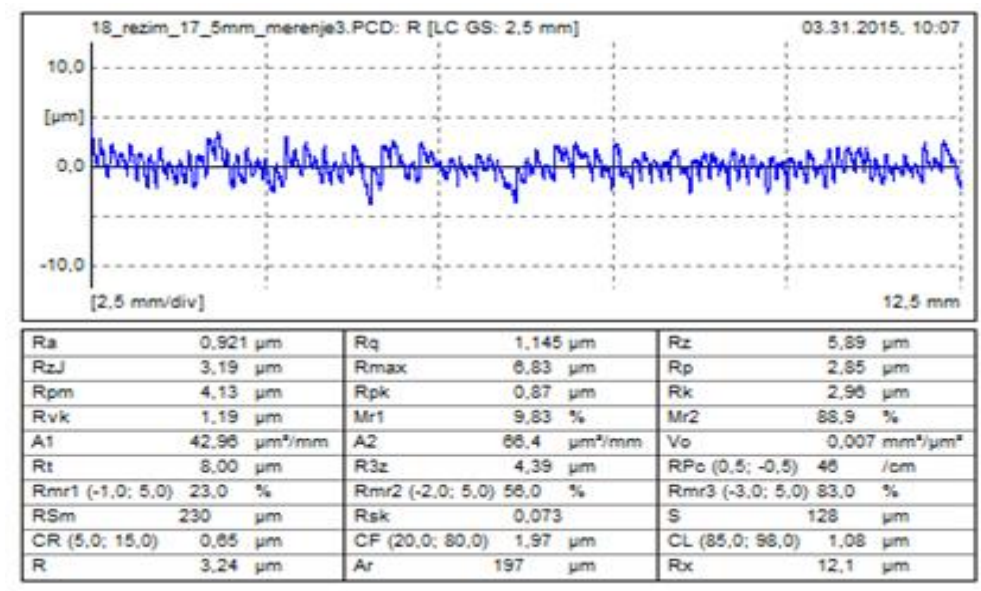

Fig. 7. The results of the measured roughness profile of machined surface

TABLE 7

Modified Table (fuzzy rules)

\begin{tabular}{|c|c|c|c|c|}
\hline \multirow[t]{2}{*}{ Ordinal number } & \multicolumn{3}{|c|}{ Code factors } & \multirow{2}{*}{$\begin{array}{l}\text { Mean. arithm. } \\
\text { roughness Ra } \mu m\end{array}$} \\
\hline & $\mathrm{v}, \mathrm{m} / \mathrm{s}$ & $\mathrm{s} 1, \mathrm{~mm} / \mathrm{t}$ & $\mathrm{a}, \mathrm{mm}$ & \\
\hline 1 & -1 & -1 & -1 & $\mathrm{C}$ \\
\hline 2 & 1 & -1 & -1 & $\mathrm{C}$ \\
\hline 3 & -1 & 1 & -1 & G \\
\hline 4 & 1 & 1 & -1 & $\mathrm{~F}$ \\
\hline 5 & -1 & -1 & 1 & $\mathrm{E}$ \\
\hline 6 & 1 & -1 & 1 & A \\
\hline 7 & -1 & 1 & 1 & $\mathrm{~F}$ \\
\hline 8 & 1 & 1 & 1 & $\mathrm{C}$ \\
\hline 9 & 0 & 0 & 0 & $\mathrm{C}$ \\
\hline 10 & $-1,41$ & 0 & 0 & $\mathrm{E}$ \\
\hline 11 & 1,41 & 0 & 0 & B \\
\hline 12 & 0 & $-1,41$ & 0 & $\mathrm{D}$ \\
\hline 13 & 0 & 1,41 & 0 & $\mathrm{H}$ \\
\hline 14 & 0 & 0 & $-1,41$ & $\mathrm{E}$ \\
\hline 15 & 0 & 0 & 1,41 & $\mathrm{H}$ \\
\hline 16 & $-1,41$ & $-1,41$ & $-1,41$ & $\mathrm{~F}$ \\
\hline 17 & $-1,41$ & 1,41 & 1,41 & I \\
\hline 18 & 0 & 1,41 & $-1,41$ & $\mathrm{~J}$ \\
\hline 19 & 1,41 & $-1,41$ & 1,41 & $\mathrm{E}$ \\
\hline 20 & 1,41 & 0 & $-1,41$ & $\mathrm{H}$ \\
\hline 21 & 1,41 & 1,41 & 0 & I \\
\hline
\end{tabular}

By selection of the first membership function carried out is its adjustment, and defines its name, so the first function membership starting on the left side will be lower, this procedure is repeated for all functions. The rules are generated as follows, for example. The rule number 1 , ac- cording to Table 7, based on the defined rules gets the following codes:

- Speed code is: $-1 \rightarrow$ low,

- Feed code is: $-1 \rightarrow$ low,

- Depth of cut code is: $-1 \rightarrow$ low. 
It is necessary to define the parameters and sets for a certain membership function. For each set defines the specific membership functions, the number of membership functions to the arithmetic mean roughness Ra is ten. For membership functions to $\mathrm{A}$, that is, for a given set of selected parameter represents the middle of the membership func- tions or his degree of membership in A is 1 . The same principle is applied to the remaining membership function for a set $B$ to the set $\mathrm{J}$.

Table 8 shows the results obtained through the fuzzy logic model (FL) with a percentage error $E$ with respect to the experimental results for $\mathrm{R}_{a}$

TABLE 8

Results obtained with the fuzzy logic model (FL) with a percentage error $\mathrm{E}$ for $\mathrm{R}$

\begin{tabular}{lllllll}
\hline \hline No. & $\mathrm{v}, \mathrm{m} / \mathrm{s}$ & $\mathrm{s} 1, \mathrm{~mm} / \mathrm{t}$ & $\mathrm{a}, \mathrm{mm}$ & $\mathrm{R}_{a} \operatorname{Exp} ., \mu m$ & $\mathrm{R}_{a} \mathrm{FL}, \mu \mathrm{m}$ & $\mathrm{E}, \%$ \\
\hline 1. & 2,93 & 0,112 & 0,75 & 0,657 & 0,67776 & 3,16 \\
2. & 4,71 & 0,112 & 0,75 & 0,666 & 0,63271 & 5 \\
3. & 2,93 & 0,177 & 0,75 & 0,909 & 0,90475 & 0,47 \\
4. & 4,71 & 0,177 & 0,75 & 0,815 & 0,82998 & 1,84 \\
5. & 2,93 & 0,112 & 1,72 & 0,765 & 0,76046 & 0,59 \\
6. & 4,71 & 0,112 & 1,72 & 0,472 & 0,45253 & 4,12 \\
7. & 2,93 & 0,177 & 1,72 & 0,869 & 0,83828 & 3,54 \\
8. & 4,71 & 0,177 & 1,72 & 0,632 & 0,63644 & 0,7 \\
9. & 3,71 & 0,141 & 1,14 & 0,604 & 0,72011 & 19,22 \\
10. & 2,35 & 0,141 & 1,14 & 0,766 & 0,85376 & 11,46 \\
11. & 5,86 & 0,141 & 1,14 & 0,505 & 0,47259 & 6,42 \\
12. & 3,71 & 0,089 & 1,14 & 0,624 & 0,56875 & 8,85 \\
13. & 3,71 & 0,223 & 1,14 & 1,048 & 1,06435 & 1,56 \\
14. & 3,71 & 0,141 & 0,5 & 0,7965 & 0,80073 & 0,53 \\
15. & 3,71 & 0,141 & 2,6 & 1,007 & 0,91490 & 9,15 \\
16. & 2,35 & 0,089 & 0,5 & 0,817 & 0,82120 & 0,51 \\
17. & 2,35 & 0,223 & 2,6 & 1,259 & 1,25800 & 0,08 \\
18. & 3,71 & 0,223 & 0,5 & 1,303 & 1,29291 & 0,77 \\
19. & 5,86 & 0,089 & 2,6 & 0,731 & 0,75600 & 3,42 \\
20. & 5,86 & 0,141 & 0,5 & 1,048 & 1,03456 & 1,28 \\
21. & 5,86 & 0,223 & 1,14 & 1,257 & 1,25798 & 0,08 \\
\hline & & & & The average error & $\Rightarrow$ & 3,94 \\
\hline \hline
\end{tabular}

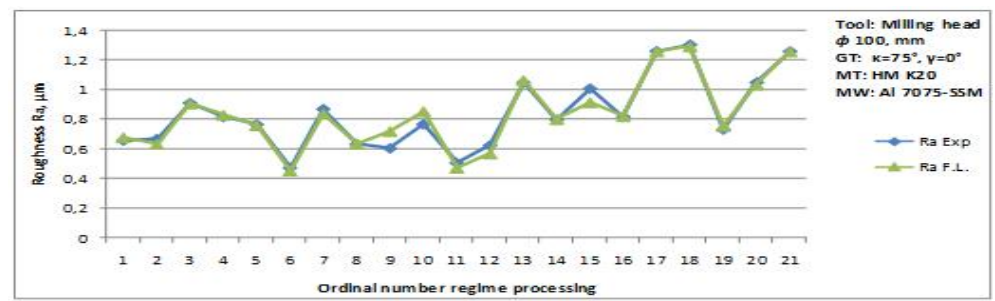

Fig. 8. Overview of the values of the arithmetic mean roughness $R_{a}$

In Figure 6 is a graph showing deviation values obtained by the fuzzy logic model (FL) values compared to the experimental values.

Table 9 shows the results obtained through the fuzzy logic model (FL) with a percentage error $E$ with respect to the experimental results for $\mathrm{R}_{\max }$. In Figure 7 is a graph showing the deviation values obtained by the fuzzy logic model (FL) compared to the experimentally obtained values. 
TABLE 9

Results obtained through the fuzzy logic model (FL) with a percentage error $\mathrm{E}$ to $\mathrm{R}_{\max }$

\begin{tabular}{lllllll}
\hline \hline No. & $\mathrm{v}, \mathrm{m} / \mathrm{s}$ & $\mathrm{s} 1, \mathrm{~mm} / \mathrm{t}$ & $\mathrm{a}, \mathrm{mm}$ & $\mathrm{R}_{\max }$ exp., $\mu \mathrm{m}$ & $\mathrm{R}_{\max } \mathrm{FL}, \mu m$ & $\mathrm{E}, \%$ \\
\hline 1. & 2,93 & 0,112 & 0,75 & 5,07 & 5,39864 & 6,48 \\
2. & 4,71 & 0,112 & 0,75 & 4,51 & 4,57950 & 1,54 \\
3. & 2,93 & 0,177 & 0,75 & 6,89 & 6,73158 & 2,3 \\
4. & 4,71 & 0,177 & 0,75 & 7,97 & 7,68362 & 3,59 \\
5. & 2,93 & 0,112 & 1,72 & 5,63 & 5,58316 & 0,83 \\
6. & 4,71 & 0,112 & 1,72 & 4,12 & 4,06184 & 1,41 \\
7. & 2,93 & 0,177 & 1,72 & 6,63 & 6,75938 & 1,95 \\
8. & 4,71 & 0,177 & 1,72 & 5,78 & 6,18796 & 7,06 \\
9. & 3,71 & 0,141 & 1,14 & 5,61 & 6,00892 & 7,11 \\
10. & 2,35 & 0,141 & 1,14 & 5,935 & 6,30717 & 6,27 \\
11. & 5,86 & 0,141 & 1,14 & 4,465 & 4,44744 & 0,39 \\
12. & 3,71 & 0,089 & 1,14 & 3,395 & 3,85784 & 13,63 \\
13. & 3,71 & 0,223 & 1,14 & 7,62 & 7,79849 & 2,34 \\
14. & 3,71 & 0,141 & 0,5 & 5,485 & 6,33326 & 15,47 \\
15. & 3,71 & 0,141 & 2,6 & 6,565 & 6,77793 & 3,24 \\
16. & 2,35 & 0,089 & 0,5 & 5,9 & 6,11099 & 3,58 \\
17. & 2,35 & 0,223 & 2,6 & 7,91 & 7,80000 & 1,39 \\
18. & 3,71 & 0,223 & 0,5 & 8,78 & 8,56661 & 2,43 \\
19. & 5,86 & 0,089 & 2,6 & 5,6 & 5,57999 & 0,36 \\
20. & 5,86 & 0,141 & 0,5 & 7,48 & 7,28395 & 2,62 \\
21. & 5,86 & 0,223 & 1,14 & 8,47 & 8,59947 & 1,53 \\
\hline & & & & The average error & $\Rightarrow$ & 4,07 \\
\hline \hline
\end{tabular}

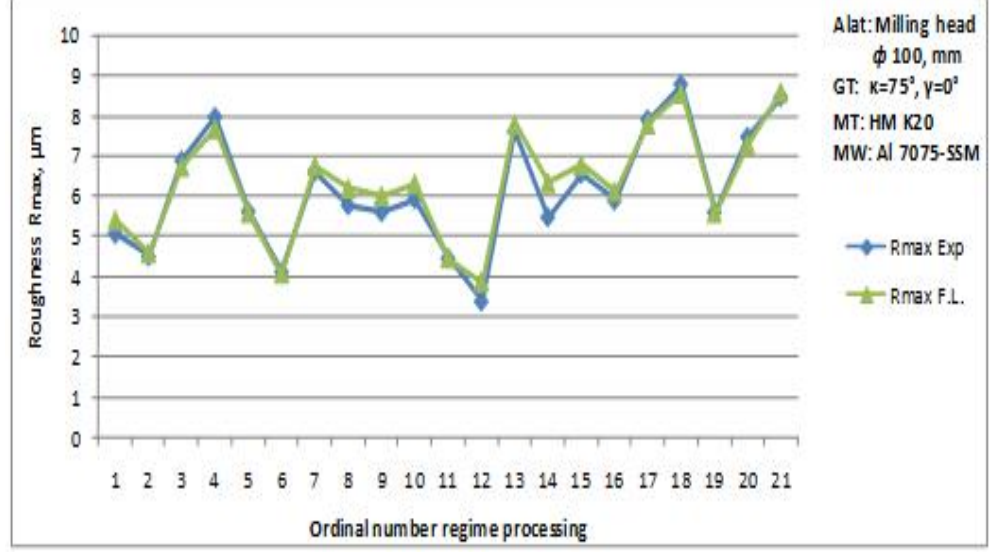

Fig. 9 . Graph of obtained values for the maximum roughness height $\mathrm{R}_{\max }$

\section{Analysis of the Results Obtained Through Different Models (DoE, FL)}

During the study, different models have been developed for calculating the value of the output characteristics of the milling process (surface roughness). In doing so, used was a variety of methods (DoE, FL). Verification of the accu- racy of those models was made on the basis of 6 additional experiments carried out according to the plan given in the table 10. During implementing, the above-mentioned models obtained these 6 results of experiment that were not used. Below is an analysis of models and variations presented in the form of the mean error. 
TABLE 10

Additional experimental results needed for model verification

\begin{tabular}{llllll}
\hline \hline Ordinal number & \multicolumn{3}{c}{ Factor } & \multicolumn{3}{c}{ Face milling } \\
& $\mathrm{v}, \mathrm{m} / \mathrm{s}$ & $\mathrm{s} 1, \mathrm{~mm} / \mathrm{t}$ & $\mathrm{a}, \mathrm{mm}$ & $\mathrm{Ra}, \mu \mathrm{m}$ & Rmax, $\mu \mathrm{m}$ \\
\hline 1 & 3,71 & 0,141 & 0,75 & 0,737 & 7,6 \\
2 & 3,71 & 0,141 & 1,72 & 0,839 & 5,07 \\
3 & 3,71 & 0,112 & 1,14 & 0,605 & 4,63 \\
4 & 3,71 & 0,177 & 1,14 & 0,709 & 5,5 \\
5 & 2,93 & 0,141 & 1,14 & 1,075 & 7,33 \\
6 & 4,71 & 0,141 & 1,14 & 0,509 & 3,97 \\
\hline \hline
\end{tabular}

To impose looks, diagrams were introduced following abbreviations for models:

- F.L - Model established based on fuzzy logic

- F.P.2 - Model obtained from the factorial plan, with the mutual influences of factors
Experimental values are shown by the abbreviation Exp.

Figure 8 shows a graph of the difference between the results obtained with the help of appropriate models for an arithmetic mean roughness $\mathrm{R}_{a}$. Comparative shows a histogram with a mean percentage error for each model.
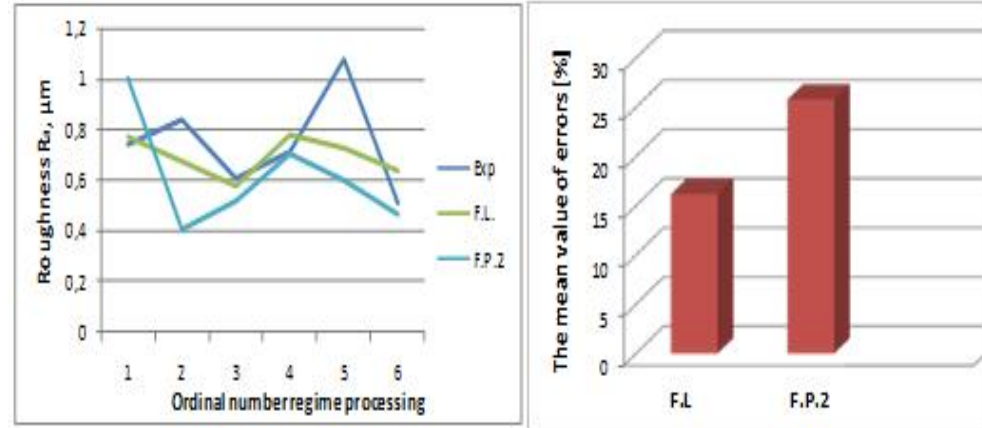

Fig. 10 . Deviations from the model experiments, the obtained mean arithmetic roughness $\mathrm{R}_{a}$ and the mean error percentages for each model

From the graph that gives smaller error tolerances established model based on fuzzy logic, while somewhat lower values give the model obtained from the factorial plan with the interaction of factors.
In figure 9 is shown a diagram with the results obtained with the help of an appropriate model for the maximum roughness height $\mathrm{R}_{\max }$ and histogram with a mean percentage error for each model.
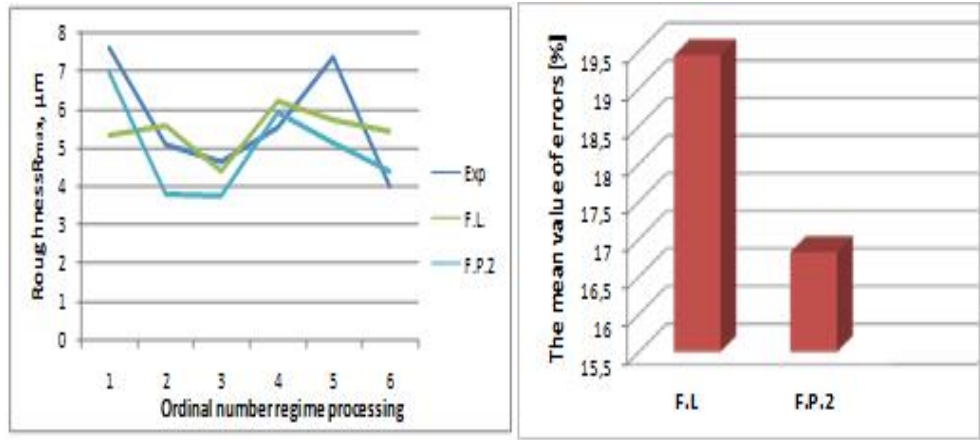

Fig. 11. Diagram of deviation from the model experiments obtained the maximum height roughness $\mathrm{R}_{\max }$ and medium error percentages for each model 
Analyzing of the diagram and histogram noticed was that for R max. A minor model deviation has been obtained in the factorial plan with the interaction of factors, while somewhat lower value gives a fuzzy logic model. But one cannot say that model of fuzzy logic did not satisfy the terms of forecasting results, because its error is below $20 \%$, which can be considered acceptable for this number of experimental data.

\section{CONCLUSION}

By the modelling of machinability functions of milling process, the parameters of the machining regime and output characteristics of the process, created were the conditions for the prediction, control and optimization of machining parameters. The modeling process is carried out with the help of mathematical models obtained on the basis of multifactor regression analysis with the help of methods based on artificial intelligence-fuzzy logic. The resulting models are comparatively analyzed and for each function machinability is proposed adoption of best model in terms of obtaining as lower as possible deviations from the experimental values. Verification of the accuracy of the model was based on additional experiments, not previously used in calculation. Featured theoretical and experimental studies have shown the applicability of new methods of modeling the processes of milling. Modeling, management and optimization of process parameters, achieved the results that will allow the prediction of the impact of certain input parameters for data constraints and the objective function. Also, the models that have been developed with the help of tools of artificial intelligence (fuzzy logic) have practical application in the industry so that research results have their own importance in this regard, and can be integrated into production systems within the machine tools with integrated memory for a knowledge base [11], [12]. This is a verified model obtained with the help of artificial intelligence, in addition to be built into the production systems that can affect computer systems based on the finite element method (FEM) to predict functions of machinability (temperature, cutting force, surface roughness and chip form).

\section{REFERENCES}

[1] P. Kardar, M. Ebrahimi, S. Bastani and M. Jalili, "Using mixture experimental design to study the effect of multifunctional acrylate monomers on UV cured epoxy acrylate resins," Progress in Organic Coatings, vol. 64, no. 1, pp. 74-80, 2009.

DOI: $10.1016 /$ j.porgcoat.2008.07.022

[2] H. Yin, Z. Chen, Z. Gu and Y. Han, "Optimization of natural fermentative medium for selenium-enriched yeast by D-optimal mixture design," LWT-Food Science and Technology, vol. 42, no. 1, pp. 327-331, 2009. DOI: $10.1016 /$ j.lwt.2008.04.002

[3] S. S. Lin, J. C. Lin and Y. K. Yang, "Optimization of mechanical characteristics of short glass fiber and polytetrafluoroethylene reinforced polycarbonate composites via D-optimal mixture design," PolymerPlastics Technology and Engineering, vol. 49, no. 2, pp. 195-203, 2010.

DOI: $10.1080 / 03602550903284297$

[4] L. C. Chen, C. M. Huang, M. C. Hsiao and F. R. Tsai, "Mixture design optimization of the composition of S, C, SnO 2-codoped TiO 2 for degradation of phenol under visible light," Chemical Engineer-

ing Journal, vol. 165, no. 2, pp. 482-489, 2010. DOI: $10.1016 /$ j.cej.2010.09.044

[5] P. Kovac, "Machining process modelling-Factorial experimental plans (in Serbian)," Master thesis, Faculty of Technical Science, University of Novi Sad, Serbia, Liman 2006.

[6] L. A. Zadeh, "Fuzzy sets," Information and Control, vol. 8, no. 3, pp. 338-353, 1965.

DOI: $10.1016 / \mathrm{S} 0019-9958(65) 90241-\mathrm{X}$

[7] D. Rodić,"Use of fuzzy logic and ANFIS systems in the investication of machining process (in Serbian)," Master thesis, Faculty of Te chnical Science, University of Novi Sad, Serbia, Liman, 2010.

[8] K. Hashmi, M. A. E. Baradie and M. Ryan, "Fuzzy logic based intelligent selection of machining parameters," Journal of Materials Processing Technology, vol. 35, no. 3, pp. 94-111, 1999.

DOI: 10.1016/S0924-0136(99)00086-2

[9] P. Kovač, D. Rodić, V. Pucovsky, B. Savković and M. Gostimirović, "Application of fuzzy logic and regression analysis for modelling surface roughness in face milling, Journal of Intelligent Manufacturing, vol. 24, no. 4, 755-762.

[10] M. Vrabel', I. Maňková, P. Kovač and J. Beňo, M. Franková and M. Palo, "A nalysis and optimization of hard turning process using Al203/TiCN ceramic TiN PVD coated insert with regard to surface roughness and cutting force components," Journal of Production Engineering, vol. 19, no. 1, pp. 22-26, 2016. 
[11] H. Maizir, R. Suryanita and H. Jingga, "Estimation of pile bearing capacity of single driven pile in sandy soil using finite element and artificial neural network methods," International Journal of Applied and Physical Sciences, vol. 2, no. 2, pp. 45-50, 2016.

DOI: 10.20469 /ijaps.2.50003-2 and S. H. Sarijo, "Artificial neural network modeling ginger rhizome extracted using rapid expansion super-critical solution (RESS) method," Journal of Advances in Technology and Engineering Research, vol. 1 , no. 1, pp. 1-14, 2015. DOI: $10.20474 /$ jater1.1.1

[12] N. A. Zainuddin, I. Norhuda, I. S. Adeib, A. N. Mustapa

— This article does not have any appendix. - 